\title{
INFLUENCE OF SMALL STEPS ON WALL PRESSURE FLUCTUATION SPECTRA MEASURED ON TU-144LL FLYING LABORATORY
}

\author{
B.M. Efimtsov, A. Yu. Golubev, TsAGI, Moscow, Russia \\ S.A. Rizzi†, NASA Langley Research Center, Hampton, VA, USA \\ A.O. Andersson, R.G. Rackl, Boeing, Seattle, WA, USA \\ E. V. Andrianov, Tupolev, Moscow, Russia
}

\begin{abstract}
Results of analyzing flight-test data of pressurefluctuation fields in front of forward-facing steps and behind backward-facing steps are presented. The range of dimensionless step heights (normalized by boundary-layer displacement thickness) in the flight test was $0.042-0.236$. The flight tests covered a mach-number range from 0.57 to 1.97 . In the machnumber ranges covered by wind-tunnel tests $(0.57$ to 0.78 and 1.5 to 2 ) data agree very well with the flight data. Very importantly, the flight-test data fill in the gap previously existing for small-step pressurefluctuations in the range of mach 0.78 to 1.5 . Increased pressure-fluctuations were observed in the transonic region.
\end{abstract}

\section{Introduction}

Forward- and backward-facing steps due to manufacturing imperfections on exterior aircraft surfaces (skin-element joints, windshield and door edges, etc.) are potential sources of aerodynamically generated interior noise. Such steps are small compared to the boundary-layer thickness. As already demonstrated in wind-tunnel experiments ${ }^{1,2}$ such steps can in flight generate local pressure-fluctuation fields with audio-frequency intensities more than one hundred times greater than those due to boundarylayer turbulence over a smooth surface. The data yielded the principal characteristics of the pressurefluctuation fields in front of forward-facing and behind backward-facing steps, random in space and time, covering useful ranges of mach, Reynolds, and Strouhal numbers, and dimensionless step height. However, a suitable transonic $(0.78<$ mach $<1.5)$ wind tunnel was not available. The mach number range of 0.8 to 1.2 is of great interest to highsubsonic-speed passenger aircraft. Even when normalized, the pressure-fluctuation intensity from these experimental data showed a tendency to ramp up as mach 0.78 was approached. Similarly, the normalized intensity at mach 1.5 was substantially greater than the ones at mach 0.78 or 2.0 . One may therefore suspect a sharp increase of pressure-fluctuation intensity in the mach-number region 0.78 to 1.5 . A flight experiment on the Tupolev 144 Flying Laboratory was carried out in order to fill in this mach-number gap ${ }^{3-4}$. Furthermore, these data extended the range of dimensionless step heights (normalized by boundary layer displacement thickness) achieved in the wind tunnel tests, to below 0.11 . This value is seen only on an aircraft's nose, whereas the behavior for much smaller values is needed for larger boundary layer thicknesses further aft. This paper presents the results of analyzing the flight-test data at small steps. The intensity distribution of spectral components of pressure fluctuations in space is investigated. Its relationships to mach, Reynolds, and Strouhal number, and dimensionless step height are compared with that obtained from the wind tunnel experiments.

\section{General information on measurements of pressure} fluctuations at steps on TU-144LL

Pressure-fluctuation fields at forward- and backward-facing steps on the Tu-144LL were realized with the use of layered metallic plates placed on the fuselage skin in two zones (Fig.1): between window apertures at a distance of $\sim 22.5 \mathrm{~m}$ and at a distance of $\sim 45 \mathrm{~m}$ from the front fuselage point. The plates were set in such a way that they partly overlapped window apertures equipped with special metallic plugs (blanks). Each of these layered metallic plates with an overall thickness of $7 \mathrm{~mm}$ consisted of two plates of smaller thickness. Directly connected with the skin was a $4 \mathrm{~mm}$ thick plate which held another $3 \mathrm{~mm}$ thick plate. The connection between them was such that the $3 \mathrm{~mm}$ plate could be easily removed. Thus, the pressure-fluctuation fields could be studied in front of forward-facing steps and behind backward facing steps of different heights $(7 \mathrm{~mm}$ and $4 \mathrm{~mm}$ ) in each of two zones on the fuselage surface.

The plates in the two zones had identical dimensions with length $758 \mathrm{~mm}$. The width of the lower ( $4 \mathrm{~mm}$ thick) plate was $305 \mathrm{~mm}$ and that of the upper ( $3 \mathrm{~mm}$ thick) plate was $216 \mathrm{~mm}$. A transition in the circumferential direction from the $216 \mathrm{~mm}$ to $305 \mathrm{~mm}$ width was made from a sculpted filler material to avoid steps in the circumferential direction. Similarly, a transition between the lower plate and the fuselage surface effectively extended the width dimension of the lower panel to $375 \mathrm{~mm}$. The plates were curved to conform to the circumferential

† AIAA Associate Fellow

Copyright (C) 2002 by The Boeing Company. Published by the American Institute of Aeronautics and Astronautics, Inc., by permission. 

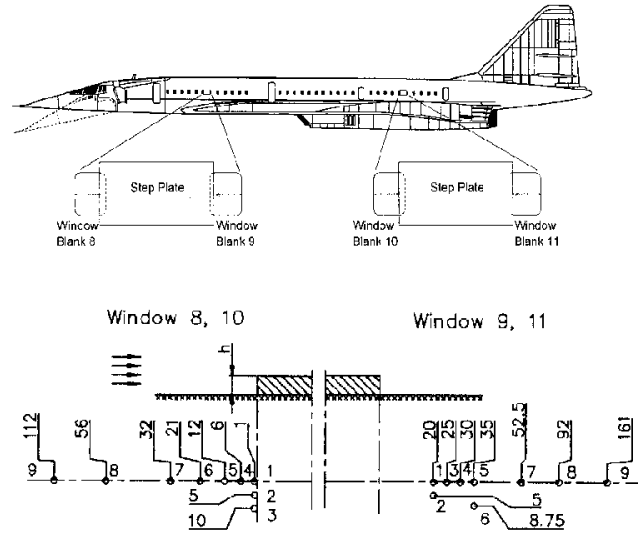

Figure 1. Disposition of window-blanks 8, 9, 10, 11 and layout of measurement points in front of forward-facing steps and behind backward-facing steps. Dimensions are in $\mathrm{mm}$.

curvature of their installed locations. There was no appreciable curvature in the longitudinal direction. While plates with even larger widths would have been desirable, this was not possible due to practical considerations by Tupolev.

The plates were mounted at different distances from the nose so that test data could be obtained at different Reynolds numbers, which differed much in the same way as the two dimensionless step heights. The experimental data obtained under such conditions permit a direct evaluation of the effects of Reynolds number and dimensionless step height

Window blanks were used in place of the windows and served as a structure through which transducers could be mounted. The window blanks had a smooth external surface and were set strictly flush with the exterior aircraft fuselage surface. The transducers for measuring pressure fluctuations in front of forward-facing steps (window blanks 8 and 10) and behind backward-facing steps (window blanks 9 and 11) were mounted through the window blanks. Details of the installation are provided in ${ }^{4}$. The sensitive elements of the transducers were strictly flush with the exterior window-blank surface. Only nine transducers were placed on each window blank; this was determined by the number of channels available in the signal-conditioning and measurement systems. It is evident that such a limited number of transducers cannot give complete information on the spatial characteristics of the extremely complex, substantially non-uniform fields of pressure fluctuations at the steps. Experimental data obtained in wind tumels ${ }^{1-2}$ were extensively used to determine transducer locations that would give maximal information about the studied fields. The locations of the pressure-fluctuation transducers (with dimensions in $\mathrm{mm}$ ) are also shown in Fig.1.
In the flow direction, the transducers were placed such that they could cover the whole zone with large pressure fluctuations for the steps of larger height $(7 \mathrm{~mm})$. To ensure direct comparison between the results at different step heights, identical dimensionless (normalized by the step height) coordinates were provided. The transducers were also set at points where the maximum pressure fluctuations were expected. Following the experimental results from $^{1-2}$, the maximum pressure fluctuations in front of forward-facing steps are observed right at the step. At backward-facing steps, the maximum pressurefluctuation intensity in wind tunnel measurements was observed approximately five step heights downstream.

In the circumferential direction, the transducers were placed only in the zone of expected maximum pressure fluctuations. Three pressure-fluctuation transducers were mounted directly in front of forward-facing steps (transducers 1-3). Four transducers were mounted behind backward-facing steps with two at a distance of $20 \mathrm{~mm}$ (transducers 1-2) and two at a distance of $35 \mathrm{~mm}$ (transducers 5-6).

The pressure-fluctuation transducers were Kulite type XCS-062-15D. These are miniature transducers, $\sim 16 \mathrm{~mm}$ in length and $1.63 \mathrm{~mm}$ in diameter. Each transducer has an external temperature-compensation module and a tube, which connects a cavity behind the sensitive element with the air space in the cabin. The sensitive element thus experiences the static pressure differential between the fuselage interior and exterior. Note however that the signal conditioning utilized a two-stage amplifier, the first of which was capacitively coupled. Thus, the DC component associated with the static pressure differential was blocked and only the fluctuating component was allowed to pass and be subsequently recorded. The sensitive element of the transducer is protected on the external side by a circular plate $(1.63 \mathrm{~mm}$ in diameter) with small circular holes through which the pressure is sensed. The holes are set in a circle of $\sim 1 \mathrm{~mm}$ diameter. The transducers were placed on windowblanks through nonmetallic threaded plugs, which provided electrical insulation from the window blanks. The protecting perforated plate and the exposed end of the insulating threaded plug were both set flush with the smooth exterior surface of the window blanks.

Calibration of the pressure-fluctuation transducers was performed with a calibrator specially developed by Boeing and able to generate a $150 \mathrm{~dB}$ sinusoidal signal at $250 \mathrm{~Hz}$, as well as broadband noise. Calibrations were performed both before and after each flight. No differences between calibrations before and after the flight were observed. Furthermore, the results of calibrations during the flight tests practically agreed with those carried out in the laboratory. 
The signal time histories from all transducers in a window-blank pair were simultaneously recorded on a Metrum RS-512 digital tape recorder. The signals were low-pass filtered at a cut-off frequency of 11.2 $\mathrm{kHz}$, equal to the upper frequency of the $1 / 3$-octave band with central frequency $10 \mathrm{kHz}$.

Measurements of pressure fluctuations at the steps were made at subsonic, supersonic and transonic velocities $0.57 \leq \mathrm{M} \leq 2.0$. In three flights, the steps were $7 \mathrm{~mm}$ high and in the last flight, the upper (3 mm thick) panels were removed so that the steps were $4 \mathrm{~mm}$ high. Measurements at subsonic and supersonic flight velocities were carried out during horizontal flight-path segments. At transonic velocities, there were relatively small variations of altitude and mach number.

After completing the program of flight tests, the data were downloaded from the Metrum recorder and written to CD-ROMs for subsequent analysis and modeling. Spectral and correlation analysis of signals was performed using the Lab Windows CVI software package

Preliminary analysis of experimental data (performed while solving methodology problems) showed that in practically all the time histories subjected to further spectral and correlation analysis, the condition of statistical steady state is fulfilled. This appeared also to be valid for the regimes of small variations of mach number. The main parts of other flight regimes were repeated in order to evaluate the stability of the experimental results. In comparable flight regimes, the differences in spectral levels were no more than $\mathrm{ldB}$. Comparison between the spectra measured in flight and on the ground with engines operating at high power allows the conclusion that pressure fluctuations at all the measurement points in flight are significantly higher than sound levels due to propulsion.

\section{Results of experimental investigations of pressure-} fluctuation spectra

As with the experiments in wind tunnels ${ }^{1,2,5}$, similarity theory was used in the present analysis. Dimensional analysis leads to the following dimensionless representation of spectral density $\Phi(\omega)$ depending on five similarity parameters:

$$
\Phi(\omega) U / q^{2} h=F\left(M, R e, h / \delta^{*}, x / h, S h\right)
$$

Here $\mathrm{U}$ is the free-flow velocity; $\mathrm{h}$ is step height; $\mathrm{q}$ is the dynamic head; $\mathrm{M}$ is the free-flow mach number, $\delta^{*}$ the boundary-layer displacement thickness; $\operatorname{Re}=\delta^{*} \mathrm{U} / \boldsymbol{v}$ is Reynolds number ( $\boldsymbol{v}$ is kinematic viscosity); $\mathrm{Sh}=\omega \mathrm{h} / \mathrm{U}$ is Strouhal number based on step height; $\mathrm{x}$ is the longitudinal distance from the step; $\omega$ is circular frequency. The dimen- sionless spectral density is presented, as usual, as a function of Strouhal number depending on parameters $\mathrm{M}, \mathrm{Re}, \mathrm{h} / \mathrm{\delta}^{*}$ and $\mathrm{x} / \mathrm{h}$. Thus, at fixed locations ( $\mathrm{x} / \mathrm{h}=$ const) the pressure-fluctuation spectra have the same similarity criteria as the averaged characteristics of flow above the step:

$$
\mathrm{M}=\text { const; } \mathrm{Re}=\text { const } \mathrm{h} / \delta^{*}=\mathrm{const}
$$

The validity of similarity condition (1) as well as the significance and completeness of the respective parameters were established in previous work ${ }^{5}$, using a forward-facing step of greater height (in comparison with the boundary-layer displacement thickness) on a plate and on the wind tunnel wall at supersonic flow velocity. The experimental results obtained in wind tunnels ${ }^{1-2}$ showed the validity of these parameters for pressure-fluctuation fields both in front of forward-facing steps and behind backward-facing steps of small dimensionless height. Unfortunately, the ranges of Reynolds number $\left(\mathrm{Re}=2.48 \times 10^{5}\right.$ $\left.6.89 \times 10^{5}\right)$ and of dimensionless step heights $\left(\mathrm{h} / \delta^{*}=0.042-0.236\right)$ in the present flight experiments were limited. Only Reynolds numbers at the upper limit of the wide range covered in the wind tunnel experiments were commensurate with those on the Tu-144LL.

The range of dimensionless step height in the Tu-144LL experiment partly overlaps the lower end of the range in wind-tunnel experiments. The minimum values of this parameter under flight conditions are about 2.5 times less than its minimum value in wind tunnels without artificial thickening of the turbulent boundary layer. The range of mach numbers realized on Tu-144LL covers practically the whole range of interest from the standpoint of noise inside high-speed airplanes.

\section{$\underline{\text { Spatial distribution of pressure-fluctuation spectra }}$}

Pressure-fluctuation fields at forward- and backward-facing steps are characterized by high intensity and possess significant spatial non-uniformity. The experimental data for the 4mm-high forwardfacing step on window blank 10 at $\mathrm{M}=0.57 ; \mathrm{M}=1.21$; $\mathrm{M}=1.97$ appear in Fig.2a-Fig.2c. The data in these figures are presented as the difference in pressurefluctuation levels $(\Delta \mathrm{L})$ in 1/3-octave frequency bands recorded at different points in front of the step and those predicted $^{6}$, i.e. the wall pressure-fluctuation levels in the same frequency bands of the turbulent boundary layer on a smooth surface with zero meanpressure gradient.

A comparison with the wind-tunnel experiments shows the same significant increase (up to $30 \mathrm{~dB}$ ) in the pressure-fluctuation intensity in front of the step, but faster reduction of the frequency region of elevated pressure fluctuations (in comparison with the 

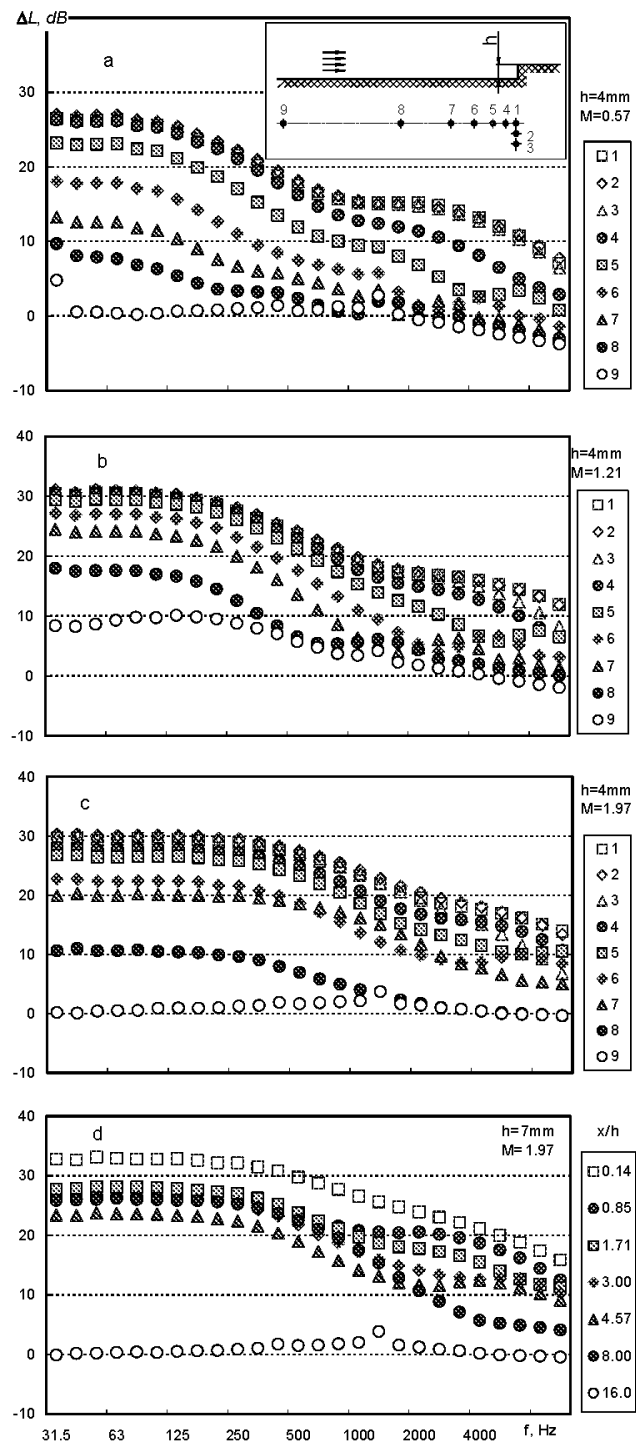

Figure 2. Spatial distribution of pressurefluctuation intensity in front of forward-facing step

turbulent boundary layer) as the observation-point distance to the step increases.
Note some features of the spatial distribution of pressure-fluctuation intensity within 1.5 step height's distance from the step. This area is (transducers 1-5) characterized by a small variation of low-frequency pressure-fluctuations. This is characteristic not only of subsonic and transonic velocities, but also of low supersonic flight velocities. As frequency increases, this feature vanishes and at frequencies above $2 \mathrm{kHz}$ we observe the same (practically equidistant) spectrum stratification as in the low-frequency region with the observation point further away from the step (at $x / h \geq 3$ ).

At low supersonic velocity (Fig.2b), substantially less spectrum stratification is observed at distances from the step up to $\mathrm{x} / \mathrm{h}=8$ (location 7 ). Nevertheless, even at location 9, the most distant from the step, the low-frequency pressure-fluctuation intensity is almost $10 \mathrm{~dB}$ higher than for a turbulent boundary layer on a smooth surface.

An unusual spectral shape at $\mathrm{M}=1.97$ (Fig.2c) is observed at point $6(\mathrm{x} / \mathrm{h}=5.25)$ and at point $7(\mathrm{x} / \mathrm{h}=8)$. This seems to be connected with the separationinduced shock located between these points. This assumption is confirmed by the pressure-fluctuation spectra presented in Fig.2d, which were obtained at a larger step height $(\mathrm{h}=7 \mathrm{~mm})$. The elevated lowfrequency levels of pressure fluctuations at location 8 $(\mathrm{x} / \mathrm{h}=8)$ are characterized by a sharper decay in the high-frequency region in comparison with the highfrequency roll-off at location $7(\mathrm{x} / \mathrm{h}=4.57)$. Probably, the shock in this case is located between locations 7 and 8 , i.e. approximately in position $\mathrm{x} / \mathrm{h} \approx 6$, which is characteristic of step heights commensurate with the boundary layer thickness 5 .

Some characteristics of the spatial distribution of pressure-fluctuation intensity in front of steps of small dimensionless height are worth noting. At small height $\left(\mathrm{h} / \delta^{*} \approx 0.066\right)$, a substantial decrease of pressure-fluctuation intensity is observed as the observation point moves away from the step (Fig.2c). We do not find as sharp a decrease of pressurefluctuation intensity behind the shock as observed at a step height commensurate with the boundary layer thickness (Fig.2d).

Fig. 3 illustrates the spatial distribution of pressure-fluctuation intensity behind backward facing steps: at the smaller step height $(\mathrm{h}=4 \mathrm{~mm})$ on window blank 11 at $\mathrm{M}=0.57$ (Fig.3a), $\mathrm{M}=1.21$ (Fig.3b) and $\mathrm{M}=1.97$ (Fig. $3 \mathrm{c}$ ). 

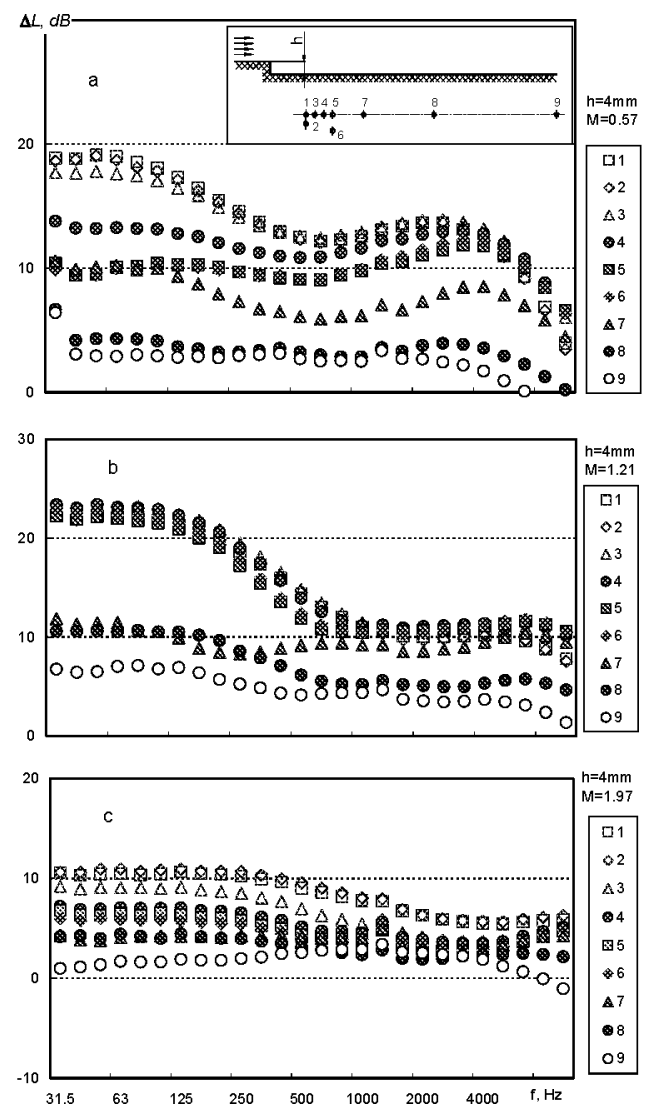

Figure 3. Spatial distribution of pressurefluctuation intensity behind backward facing step.

These data, as well as the experimental data obtained in wind tunnels ${ }^{2}$ show that behind a backwardfacing step the region of increased pressure fluctuations is of substantially larger extent than that observed in front of forward-facing steps. As the observation point moves away from the backward-facing step, the maximum values of $\Delta \mathrm{L}$ and their variation in the high-frequency part of the spectrum also agree with those observed in the wind tunnel experiments at $\mathrm{M} \approx 0.5$ and $M \approx 2$. However, at low frequencies, the values of $\Delta \mathrm{L}$ at points 1-3 obtained in flight experiments are significantly higher than those observed at $\mathrm{M} \approx 0.5$ in the wind tunnel experiment.

The characteristics of low-frequency pressurefluctuation behavior revealed in the flight experiment can be explained only by additional disturbances. The only supposition that can be made is that these are generated by the interaction of the flow with the front and side surfaces of the plate. This supposition is supported in a way by the correlations between the spectral components of the pressure-fluctuation field between a point directly in front of the plate and a point behind it, where attachment of the flow separated at the rear edge of the plate was expected. Con- sidering this, the results of measurements behind a backward facing step on Tu-144LL are, strictly speaking, valid only at high frequencies, and influenced by the finite extent of the step plates at lower frequencies.

For the spectra in $1 / 3$-octave frequency bands, measured behind the step in the zone of flow attachment at low subsonic flight velocity, a gently sloping maximum is characteristic in the high-frequency region (Fig.3a). It also manifests itself behind the attachment region downstream. A similar maximum was also observed in the wind tunnel experiment. As mach number increases, this spectral feature decreases and at $\mathrm{M}=1.5$ it is no longer observed.

Let us note another observation of the measurement results behind a backward-facing step on the flying laboratory regarding the position of the zone of the separated-boundary-layer reattachment. Precisely in this zone the maximum intensity of pressure fluctuations is observed at practically all frequencies. Fig.4 illustrates a variation of the position of maximum pressure fluctuations behind the step as the flight mach number increases. At $\mathrm{M}=0.57$ the maximum position approximately corresponds to $\mathrm{x} / \mathrm{h}=5.0$. At $\mathrm{M} \sim 1.0$, the maximum intensity position corresponds $x / h=7.5$. Further increase of the mach number results in a reverse shift of the maximum, and at $\mathrm{M}=1.96$ its shift to the neighboring measurement point located at $\mathrm{x} / \mathrm{h}=4.3$ is quite evident.

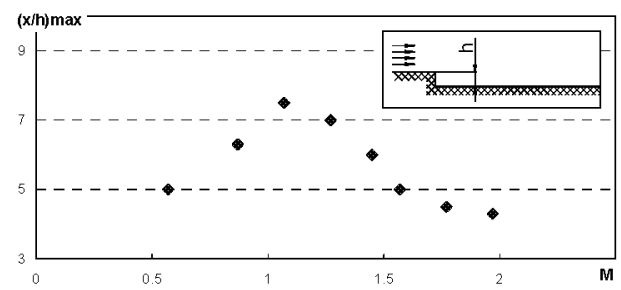

Figure 4. Shift of the intensity maximum of pressure fluctuations behind backward-facing step at different mach numbers.

One source of additional uncertainty is flow downwash. The flow visualization data on Tu-144 and directly on Tu-144LL provide evidence ${ }^{4}$ of the presence of pronounced downwashes in the measurement zones. The most intensive downwashes were observed in the first zone located closer to the fuselage nose (on window-blanks 8 and 9).

The effect of flow downwash on pressurefluctuations at steps remains unstudied at present. However, it is well known that downwashes disturb the flow's two-dimensionality, which can lead to changes in the wall pressure-fluctuation intensity of separated flows. As a rule, three-dimensional effects in two-dimensional separated flow cause some weakening of wall pressure fluctuations. 
Effect of Reynolds number on pressure-fluctuation spectra

The effect of Reynolds number on the pressure-fluctuation spectra in front of forward-facing steps was determined by comparing the dependencies of dimensionless spectral density on Strouhal number (1) at slightly different values of dimensionless step height (at $\mathrm{h}=7 \mathrm{~mm}$ on window blank 10 and at $\mathrm{h}=4 \mathrm{~mm}$ on window blank 8 ). This was done at different measurement points with identical dimensionless coordinates $\mathrm{x} / \mathrm{h}$ successively for each mach number. Here, as an illustration, the data on such a comparison of spectra are presented only for $\mathrm{M}=0.57$ (Fig.5a), $\mathrm{M}=1.2$ (Fig. 5b) and $\mathrm{M}=1.97$ (Fig. 5c). In the measurement zone located closer to the nose (window-blank 8) at $\mathrm{M}=0.57$ the pressure-fluctuation intensity in front of the step is lower than in the zone located downstream (window-blank 10). The effect decreases at $\mathrm{M}=1.2$ (Fig.5b) and at $\mathrm{M}=1.97$ (Fig.5c) it is practically not manifested.
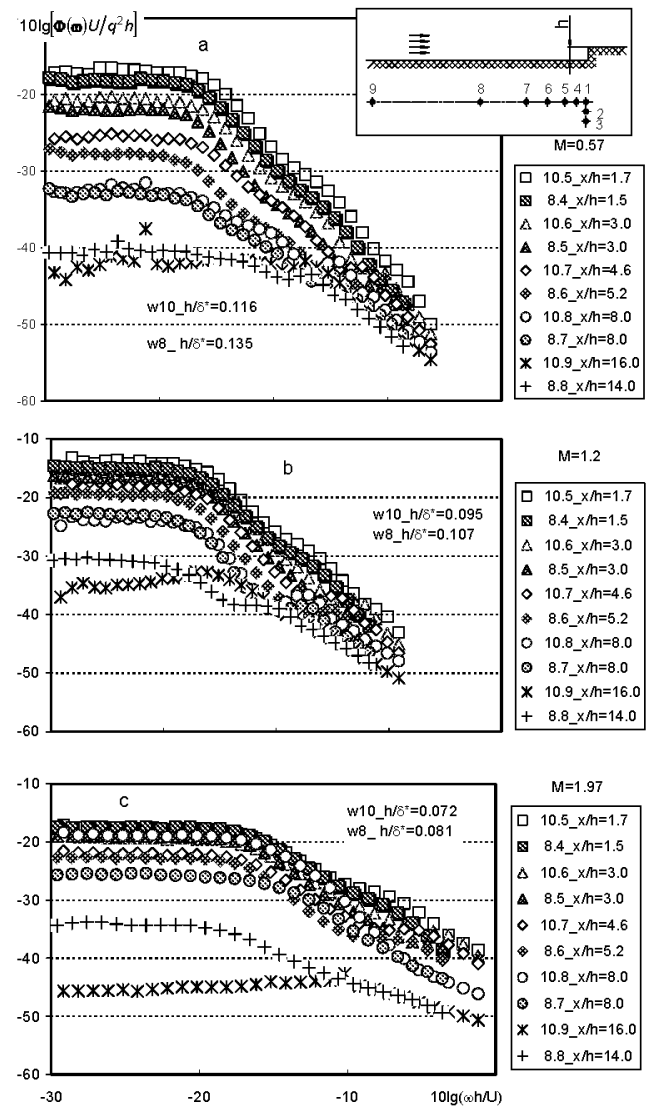

Figure 5. Effect of Reynolds number on pressurefluctuation spectra in front of forward-facing step.

Under flight conditions of Tu-144LL corresponding to $\mathrm{M}=1.2$ the flow visualization indicates significantly weaker flow downwashes than at $\mathrm{M}=0.57$. At $\mathrm{M} \approx 2.0$ the downwashes are minimal and weak. Thus, the difference observed in the decrease of the pressure-fluctuation intensity in front of the step in the zone of window blank 8 at different mach numbers appears to correlate with the observed downwashes in this zone, and is not a function of Reynolds number. Such a conclusion is also confirmed by the results of pressure-fluctuation investigations in front of a forward-facing step obtained in the wind-tunnel experiments ${ }^{1}$, which demonstrated a very small effect of Reynolds number over a wide range up to the values close to the minimum values encountered on Tu-144LL

In evaluating the effect of Reynolds number on pressure fluctuations behind backward-facing steps, low-frequency spectrum components will not be used for reasons previously cited, especially in the region of the separated-flow reattachment (at $3 \leq x / h \leq 8$ ). In the region of high Strouhal numbers one can observe much the same difference of the dimensionless spectral density of pressure fluctuations (Figs.6a,b) in the first (window-blank 9) and the second (windowblank 11) zones, as in front of the steps (Figs.5a-c). This difference can also be explained by flow downwashes, and not by a Reynolds number effect, which was not observed in the wind tunnel experiments either
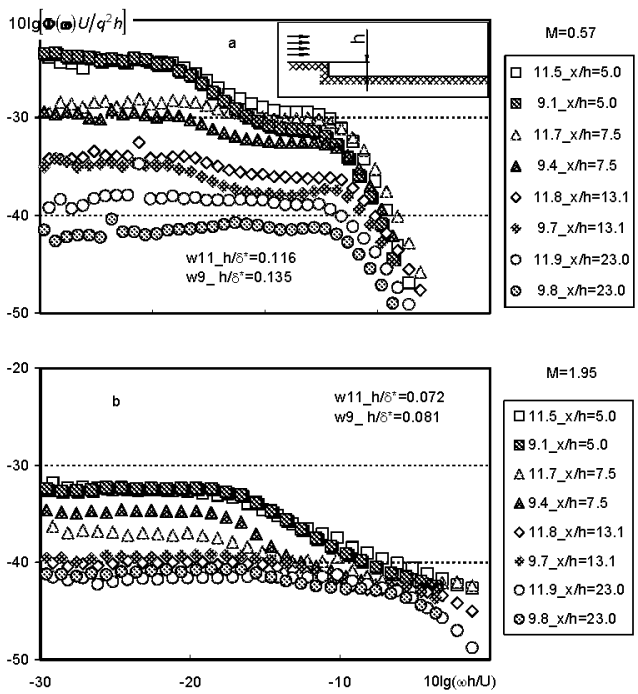

Figure 6. Effect of Reynolds number on pressurefluctuation spectra behind backward-facing step.

\section{Effect of dimensionless step height on pressure- fluctuation spectra}

Fig.7a illustrates the effect of dimensionless height immediately in front of a forward-facing step on pressure fluctuation spectra for $\mathrm{M}=0.87$. At small Strouhal numbers, the effect of dimensionless step 


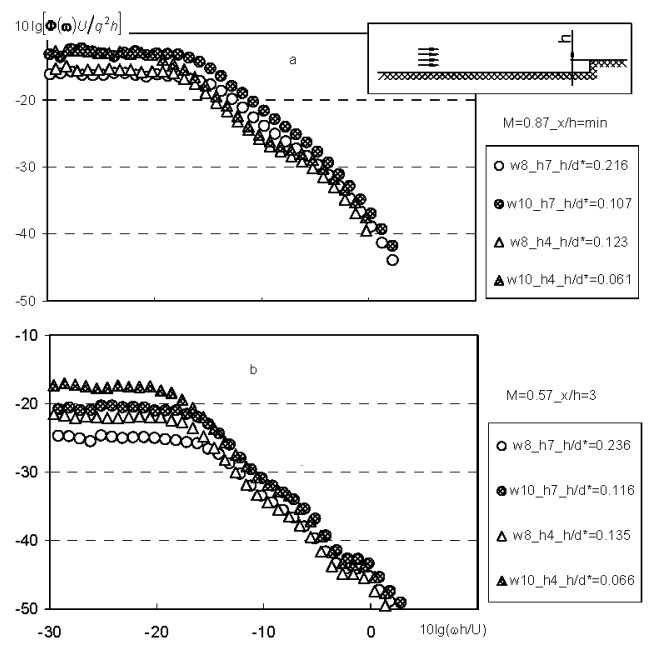

Figure 7. Effect of dimensionless step height on pressure-fluctuation spectra in front of forwardfacing step.

height appears to be zero. In the region of Strouhal numbers, where a decrease in dimensionless spectral density is observed, one can clearly see stratification of dimensionless spectra relating to different values of $\mathrm{h} / \delta^{*}$, principally due to the variation in cut-off frequency (transition from constant intensity $\left[\mathrm{Sh}^{\circ}=\right.$ Strouhal to power zero] to rolling off with frequency). In this case, a higher cut-off frequency corresponds to a larger value of $h / \delta^{*}$. Precisely such effects of dimensionless step height (only with a lower cut-off frequency) were revealed in the wind tunnel experiments ${ }^{1}$. These effects are observed at different mach numbers.

As the observation point moves upstream from a forward-facing step, the spectrum quickly changes character. Already at $\mathrm{x} / \mathrm{h}=3$ (Fig. $7 \mathrm{~b}$ ) a reverse picture is observed: the dimensionless spectra are stratified in the region of small Strouhal numbers (in the area of dependence $\sim \mathrm{Sh}^{\circ}$ ) and differ little in the area of spectral rolloff at higher frequencies. This picture remains practically unchanged as the observation point moves away from the step. Although not shown, it is invariant with respect to mach number, until the observation point falls in the vicinity of the separation-induced shock. Such a transformation of spectra was indeed observed in the wind-tunnel experiments.

Evaluation of the effect of dimensionless height on the pressure-fluctuation spectra, measured behind a backward-facing step, is worthwhile only for the region of high Strouhal numbers where the experimental data are not influenced by the finite step-plate size. Only some tendencies related to the effect of dimensionless step height can be reported here: increase of $h / \delta^{*}$ leads to some increase of spectral den- sity in this Strouhal-number region at practically all mach numbers and to some increase of cut-off frequency at supersonic flight conditions. The latter point is illustrated by the experimental data at $\mathrm{M}=1.97$ (Fig.8).

Effect of mach number on pressure-fluctuation spectra

In the experiment on $\mathrm{Tu}-144 \mathrm{LL}$, the range $0.57 \leq \mathrm{M} \leq 1.97$ was covered. Sufficiently reliable data on the mach-number effect on pressure fluctuations in front of a forward-facing step over the whole range of Strouhal numbers of practical interest were obtained. Unfortunately, only the experimental data obtained in the range of high Strouhal numbers, can be considered valid for pressure fluctuations behind backward-facing steps.

The mach-number effect on the pressurefluctuation spectra directly in front of the step ( $\mathrm{x} / \mathrm{h}=\mathrm{min}$ ) on window-blank 10 at $\mathrm{h}=4 \mathrm{~mm}$ is illustrated in Fig.9. It is clearly seen that the mach-

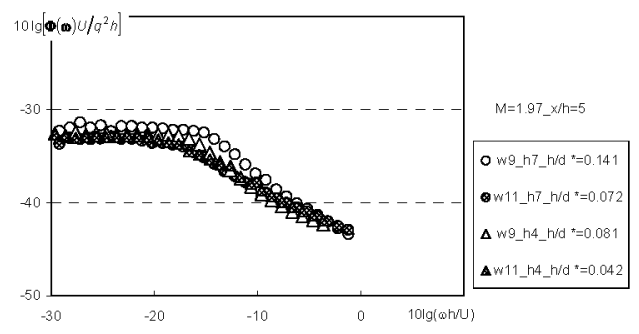

Figure 8. Effect of dimensionless step height on pressure-fluctuation spectra behind backward-facing step.

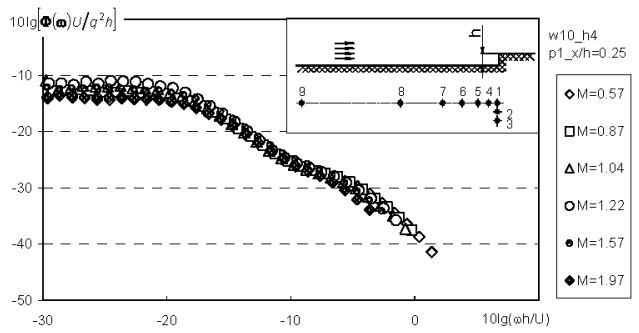

Figure 9. Effect of mach number on pressurefluctuation spectra in front of forward-facing step.

number effect is more pronounced in the range of low Strouhal numbers where dimensionless spectral density is observed to be independent of Strouhal number. The maximum values of dimensionless spectral density in this case correspond to low supersonic flight velocities $(\mathrm{M}=1.22)$.

Fig. 10 presents the dependence of dimensionless spectral density on mach number at fixed Strouhal number corresponding to the region where the spectral density is independent of Strouhal number. In essence, this is the dependence of the principal pa- 
rameter of the prediction model of the pressurefluctuation field in front of a forward-facing step, indicated in reference ${ }^{1}$ as $\mathrm{F}_{\text {mo }}$ (see section 3 ).

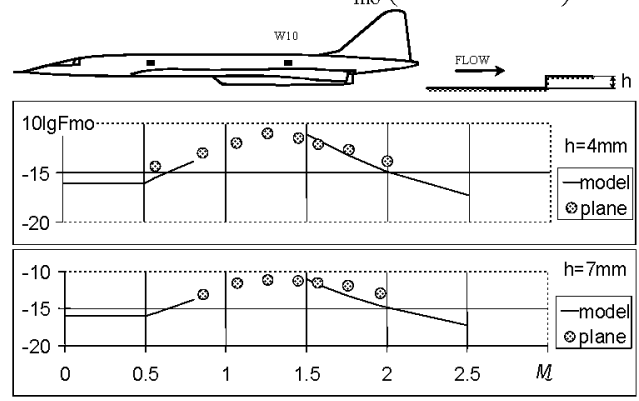

Figure 10. Effect of mach number on maximum intensity of pressure fluctuations in front of forwardfacing step.

Fig.10 also presents the dependence of $F_{m o}$ on mach number, as obtained from wind-tunnel experiments and used in the development of the prediction model ${ }^{1}$ of the characteristics of the pressure-fluctuation field in front of a forward-facing step of small height. In the ranges of mach number covered by wind-tunnel experiments, the flight experiment confirms the reliability the prediction model. Furthermore, the experiment on Tu-144LL permits extending this prediction model to a parameter region heretofore not studied: $0.78 \leq \mathrm{M} \leq 1.5$.

If displayed in 1/3-octave bands, the spectrum for a backward-facing step shows a marked peak at high frequencies for low mach numbers (Fig.11). At higher mach numbers, this peak disappears. This behavior is evident in the mach-number range 0.87 1.57 not previously covered in wind-tunnel experiments. This is why the prediction model $^{2}$ presents different relations for evaluating pressure fluctuations behind backward facing steps for subsonic $(\mathrm{M} \leq 0.78)$ and supersonic $(\mathrm{M} \geq 1.5)$ flows. The new experimental material permits these two relations to be joined.

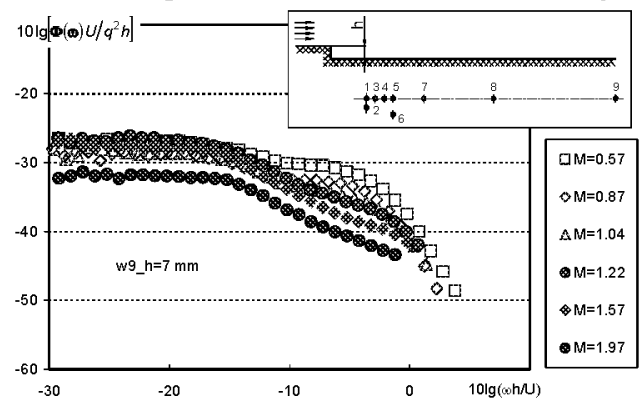

Figure 11. Effect of mach number on pressurefluctuation spectra behind backward-facing step.

The low-frequency part of the spectrum in Fig. 11 for $M \leq 1.5$ seems to be distorted by the addi- tional disturbances from the plate discussed above. However, the spectrum behavior at $\mathrm{M}=1.57$ and $\mathrm{M}=1.97$ characterized by their practically equidistant stratification indicates that the experimental data at low frequencies, obtained at $\mathrm{M}=1.97$ on window blank 9 at $\mathrm{h}=7 \mathrm{~mm}$, possibly is reliable.

\section{Modeling of the experimental data}

The prediction models for pressure fuctuations at forward-facing steps ${ }^{1}$ and backward-facing steps $s^{2}$ can now be extended to lower non-dimensional step heights $\left(\mathrm{h} / \delta^{*}<0.3\right)$ and to the transonic region based on the flight data. The form of these prediction models is

$$
\Phi(\mathrm{x}, \omega)=\frac{\mathrm{q}^{2} \mathrm{~h}}{\mathrm{U}} \mathrm{F}_{\mathrm{m}} \Psi(\mathrm{M}) \exp \left(-\left|\mathrm{x}-\mathrm{x}_{\mathrm{m}}\right| / \mathrm{L}\right)+\Phi_{\mathrm{b} \ell}(\omega)
$$

where the factor $F_{m}$ describes the Strouhal-number dependence. For a forward-facing step

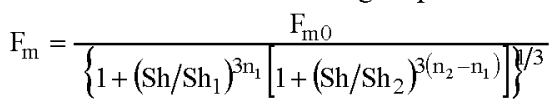

The characteristic parameters $\left(\mathrm{Sh}_{2}, \mathrm{n}_{1}, \mathrm{n}_{2}\right)$ remain unchanged while $\mathrm{Sh}_{1}, \mathrm{~F}_{\text {mo }}$ take on new values:

$$
\begin{gathered}
\mathrm{F}_{\mathrm{m} 0}=0.035 ; \\
\mathrm{Sh}_{1}=\Psi_{1}(\mathrm{M})\left\{\begin{array}{l}
0.148\left(\mathrm{~h} / \delta^{*}\right) \text { at } 0.042 \leq \mathrm{h} / \delta^{*} \leq 0.11 ; \\
0.13 \mathrm{l}\left(\mathrm{h} / \delta^{*}-0.11\right)+0.01628
\end{array}\right. \\
\Psi_{1}(\mathrm{M})= \begin{cases}1 & \text { at } 0.11<\mathrm{h} / \delta^{*} \leq 0.3 ; \\
1+(\mathrm{M}-0.8) 0.8 & \text { at } \quad \mathrm{M} \leq 0.8 ;\end{cases}
\end{gathered}
$$

Dependence of the pressure-fluctuation spectral density on mach number is described by a modified empirical dependence extended to the whole machnumber region $(\mathrm{M} \leq 2)$.

$$
\Psi(\mathrm{M})=\left\{\begin{array}{cl}
1 & \text { at } \mathrm{M} \leq 0.5 \\
\frac{4(\mathrm{M} / 1.4)^{1.32}}{1+(\mathrm{M} / 1.4)^{4}} & \text { at } 0.5<\mathrm{M} \leq 2
\end{array}\right.
$$

The non-uniformity scale of the field is determined in the same way as for prediction model ${ }^{1}$, and is given as an empirical dependence of dimensionless step height and mach number:

In the region of $0.042 \leq \mathrm{h} / \delta^{*} \leq 0.15$

$$
\begin{aligned}
& \mathrm{L} / \mathrm{h}=\max \left\{\frac{\overline{\mathrm{L}}_{0} \boldsymbol{\Psi}(\mathrm{M})}{1+2.5 \mathrm{Sh}}, 2\right\} \\
& \left.\overline{\mathrm{L}}_{0}=0.65 \mathrm{~h} / \delta^{*}\right)^{-0.625}
\end{aligned}
$$

In the region $0.15<\mathrm{h} / \delta^{*} \leq 0.3, \mathrm{~L} / \mathrm{h}=\overline{\mathrm{L}}_{\circ} \Psi(\mathrm{M})$.

Improvement of the prediction model for the pressure-fluctuation field behind a backward-facing step offered the opportunity to join different dependencies for spectral density at subsonic and supersonic flight velocities. A superposition of two fields with 
different dependencies of spectral density on mach number was used. The typical dimensionless spectrum of the first field $\left(\mathrm{F}_{\mathrm{m}}\right)$ is described by the same relation as the one used in the prediction model ${ }^{2}$ with modified characteristic parameters:

$$
\begin{gathered}
\mathrm{F}_{\mathrm{m}}=\frac{\mathrm{F}_{\mathrm{m} 0}}{\left[\left(\mathrm{Sh} / \mathrm{Sh}_{1}\right)^{2 \mathrm{n}_{1}}+\left(\mathrm{Sh} / \mathrm{Sh}_{2}\right)^{2 \mathrm{n}_{2}}\right]^{\mathrm{b} / 2}} \\
\mathrm{~F}_{\mathrm{m} 0}=\left\{\begin{array}{l}
11 \times 10^{-4} \text { at } 0.042 \leq \mathrm{h} / \delta^{*} \leq 0.07 ; \\
{\left[11+20\left(\mathrm{~h} / \delta^{*}-0.07\right)\right] 10^{-4} \text { at } 0.07<\mathrm{h} / \delta^{*} \leq 0.3 ;}
\end{array}\right. \\
\mathrm{Sh}_{1}=10^{-3} ; \\
\mathrm{Sh}_{2}=\left\{\begin{array}{l}
0.02 \text { at } 0.042 \leq \mathrm{h} / \delta^{*} \leq 0.07 ; \\
0.02+0.08\left(\mathrm{~h} / \delta^{*}-0.07\right) \text { at } 0.07<\mathrm{h} / \delta^{*} \leq 0.3 ;
\end{array}\right.
\end{gathered}
$$$$
\text { Dependence of pressure-fluctuation intensity }
$$
on mach number for the first field is described by the following empirical relations:

$$
\Psi(M)=\left\{\begin{array}{cc}
1 & \text { at } M \leq 0.75 \\
1+2(M-0.75) & \text { at } 0.75<M \leq 1 \\
1.5 & \text { at } 1<M \leq 1.5 \\
1.5-2(M-1.5) & \text { at } 1.5<M \leq 2
\end{array}\right.
$$

The typical dimensionless spectrum for the second field (quantities with *) is presented in the form:

$$
\mathrm{F}_{\mathrm{m}}^{*}=\frac{\mathrm{F}_{\mathrm{mo}}^{*}\left(\mathrm{Sh} / \mathrm{Sh}_{1}^{*}\right)^{\mathrm{n}_{1}^{*}}}{1+\left(\mathrm{Sh} / \mathrm{Sh}_{1}^{*}\right)^{\mathrm{n}_{2}^{*}}}
$$

The characteristic parameters corresponding to these fields are described by the relations:

$$
\begin{array}{r}
\mathrm{F}_{\mathrm{m} 0}^{*}=\mathrm{F}_{\mathrm{m} 0} ; \mathrm{Sh}_{1}^{*}=0.5 ; \\
\mathrm{n}_{1}^{*}=0.2 ; \mathrm{n}_{2}^{*}=3.0
\end{array}
$$

Dependence of pressure fluctuations on mach number for the second field is described by relations indicating its vanishing effect as $\mathrm{M}$ approaches 1.5 .

$$
\Psi^{*}(\mathrm{M})=\left\{\begin{array}{lr}
1 & \text { at } \mathrm{M} \leq 0.5 \\
1-(\mathrm{M}-0.5) & \text { at } 0.5<\mathrm{M} \leq 1.5 \\
0 & \text { at } 1.5<\mathrm{M} \leq 2
\end{array}\right.
$$

The new experimental data also permit refining the location of the pressure-fluctuation intensity maximum:

$$
(\mathrm{x} / \mathrm{h})_{\max }=\left\{\begin{array}{cc}
5 & \text { at } \mathrm{M} \leq 0.5 \\
5+5(\mathrm{M}-0.5) & \text { at } 0.5<\mathrm{M} \leq 1 \\
7.5 & \text { at } 1<\mathrm{M} \leq 1.4 \\
7.5-6(\mathrm{M}-1.4) & \text { at } 1.4<\mathrm{M} \leq 2
\end{array}\right.
$$

The non-uniformity scale of the pressurefluctuation field is unmodified from the prediction model $^{2}$.

\section{Concluding Remarks}

Spectral analysis of the results of pressurefluctuation measurements in front of forward-facing steps and behind backward-facing steps showed that in the flight experiment on Tu-144LL a great body of experimental data of practical interest was obtained. These data confirm mainly the reliability of the windtunnel experimental results ${ }^{1-2}$ from which the prediction model is built. Moreover, they permit improving this model and its extension to those regions of real parameters, which were not covered in the wind tunnel experiments. The case in point here is the region of mach numbers $(0.78<\mathrm{M}<1.5)$ and the region of small dimensionless (normalized by the boundary layer displacement thickness) step heights $\left(\mathrm{h} / \delta^{*}<0.11\right)$ for which reliable experimental data were not obtained in wind tunnels. Exactly this mach-number region, its lower part $(0.78<\mathrm{M} \leq 1.2)$ particularly, is of greatest interest for high-speed aircraft.

\section{Acknowledgements}

The authors want to thank the Boeing Company for financial support of this work and in particular S.V. Kravchenko and D.H. Reed for helpful technical discussions and organization of the work.

\section{References}

1. Efimtsov B.M. Kozlov N.M. Kravchenko S.V. Andersson A.O. "Wall pressure-fluctuation spectra at small forward-facing steps". AIAA-991964.

2. Efimtsov B.M. Kozlov N.M. Kravchenko S.V. Andersson A.O. "Wall pressure-fluctuation spectra at small backward-facing steps". AIAA-20002053.

3. Rizzi S.A., Rackl R.G. and Andrianov E.V., "Flight test measurements from the Tu-144LL structure/cabin noise experiment," NASA/TM2000-209858, January 2000.

4. Rizzi S.A., Rackl R.G. and Andrianov E.V., "Flight test measurements from the Tu-144LL structure/cabin noise follow-on experiment," NASA/TM-2000-209859, February 2000.

5. Efimtsov B.M., Kuznetsov V.B. "Wall pressure fluctuation spectra at supersonic streamline of a forward facing step". Uchenye Zapiski TsAGI, v.20, No.3, 1989.

6. Efimtsov B.M. "Similarity criteria of wall pressure fluctuation spectra of the turbulent boundary layer". Akustichesky Zhurnal, v.30, ed.1, 1984. 